\title{
A Method for Dimensioning Micro-Scale Solar Energy Harvesting Systems Based on Energy Level Simulations
}

\author{
Sebastian Bader*, Torsten Schölzel ${ }^{\dagger}$, Bengt Oelmann* \\ *Department of Information Technology and Media \\ Mid Sweden University, Sundsvall, Sweden \\ Email: \{sebastian.bader, bengt.oelmann\}@miun.se \\ †SOLARWATT AG, Dresden, Germany \\ Email: torsten.schoelzel@solarwatt.de
}

\begin{abstract}
Solar energy harvesting gains more and more attention in the field of wireless sensor networks. In situations, where these sensor systems are deployed outdoors, powering sensor nodes by solar energy becomes a suitable alternative to the traditional way of battery power supplies. Since solar energy, opposed to batteries, can be considered as an inexhaustible energy source, scavenging this source allows longer system lifetimes and brings wireless sensor networks closer to be an autonomous system with perpetual lifetime. Despite the possibility of designing and constructing these harvesting system, dimensioning becomes a crucial task to fit implemented components to application and load system demands. In this paper we present a way of dimensioning solar harvesting systems based on simulation. Method and implementation of component and system models are described on the basis of an example architecture that has been used in prior work. Furthermore we evaluate the model in comparison to deployment of the same architecture and show the suitability of using the simulation as a support to optimize choices for system parameters.
\end{abstract}

Keywords-energy harvesting; wireless sensor networks; solar radiation; modeling; dimensioning; availability simulation;

\section{INTRODUCTION}

In the last decade wireless sensor networks have been demonstrated to be a feasible measurement technology in many applications. Besides having the possibility of offering immensely reduced installation costs, wireless sensor networks gain interest in application areas, where autonomous and large-scale observations are desired. In environmental monitoring systems, as one of these application areas, wireless sensor networks can cover large areas of interest and therefore consist of many sensor nodes. Replacing batteries in these situations is inconvenient and antagonizes the initial advantages of using wireless sensor networks as an autonomous measurement system.

With the decreasing power consumption due to advances in integrated circuits and energy-efficient communication protocols, energy harvesting becomes a mechanism towards perpetual energy supply in these systems. Typical ambient energy sources under consideration are solar, wind, vibration and temperature difference. Due to availability, existing conversion solutions and energy levels, solar energy harvesting gained most attention in outdoor applications so far.

Although solar radiation is usually more predictable for a given location and time than for example wind, spatial and temporal distribution shows strong variations. Therefore the design, and particularly the dimensioning, of the harvesting system is tremendously dependent on the application and deployment-site of the system. However, this task is rather complex. Even if a harvesting architecture has been chosen according to application requirements, dimensioning parameters such as needed solar panel size, storage capacity or possible load consumption (i.e. most of the time the node's duty-cycle) are difficult to predict for a targeted location.

In this paper, energy level simulation is proposed as a method for dimensioning solar energy harvesting systems. The simulator comprehends three parts, the architectural model of the harvester and load system, the environmental input parameter (i.e., primarily irradiance levels) and the simulation condition (e.g., sweep of panel size). We evaluate this method with an example architecture presented in [1] and irradiance data, measured with high temporal resolution. Further, we validate the system model in comparison to deployment results and demonstrate the simulator's capabilities for dimensioning tasks of the same architecture.

\section{RELATED WORK}

There have been several systems for solar energy harvesting for wireless sensor networks or small embedded systems in literature. Some well known include, but are not limited to, those presented in [2]-[5]. The presented architectures and implementations have demonstrated feasibility of using solar energy for powering sensor nodes in outdoor deployments. Furthermore different architectural choices have been shown, each representing different application constraints or optimization goals. Variations cover different storage technologies, such as rechargeable batteries [2], supercapacitors [3] or combinations [5], different control mechanisms (e.g. software-based [5] or hardwarebased [2] solutions) and optimizations for performance [3] or minimum energy overhead [1]. Although these systems have been shown functioning in implementations at least for 
certain applications or locations, dimensioning of respective architectures for different situations was rarely addressed.

Modeling of key components in solar energy harvesting systems has been addressed in research independently to wireless sensor network application. A well established model for solar panels is described in [6], [7] and further, a more detailed modeling of internal cell operation has been covered in [8]. When it comes to energy storage, battery modeling has been addressed in [9] and a double layer capacitor model is proposed in [10]. In [11] a general overview of energy device modeling and combination is given.

Research on modeling complete solar energy harvesting systems is much more limited. Work related to this topic was addressed in [9], [12], [13]. In [12] analytical prediction of harvested energy and consequence for battery charge level is examined. However, the presented results show that the model is not yet accurate enough to make decisions on system scaling. The simulation tool presented in [13] addresses a similar problem as this paper does, namely sizing of modules, but concentrates on larger sized harvesting systems, which leads to different approaches and optimization goals. Jeong describes modeling and simulation of micro-scale solar power systems in [9]. The prime goal of this system, however, seems to be architecture validation rather than dimensioning of a given architecture. A subset of the work described by Jeong was published in [14]. This work addresses also capacity planning of micro solar energy harvesting systems, however, does this rather analytically.

Opposed to that, we propose energy level simulations as a method for dimensioning components, such as solar panel size or storage capacity, based on application and location constraints. We utilize a hybrid modeling approach, using detailed component representation and abstractive functions in the same system model. This approach allows to reach accuracy where needed, while enabling fast implementation time otherwise. The simulation method we present supports dimensioning decisions and system optimizations.

\section{THEORY}

A typical solar energy harvesting system consists of solar energy conversion, energy storage, energy management and load system. However, the way these modules are implemented, and how they are connected can vary tremendously and is usually dependent on the application (i.e. load demands, location and time-span). The modeling method and simulations are based on an example architecture, presented in [1]. This architecture was chosen due to familiarity with the system and existing deployment results. Furthermore it is very flexible towards changes and additions. Nevertheless, any other architecture could be modeled in a similar fashion as described below. In the following the example architecture, as well as its component and system models are presented in more detail.

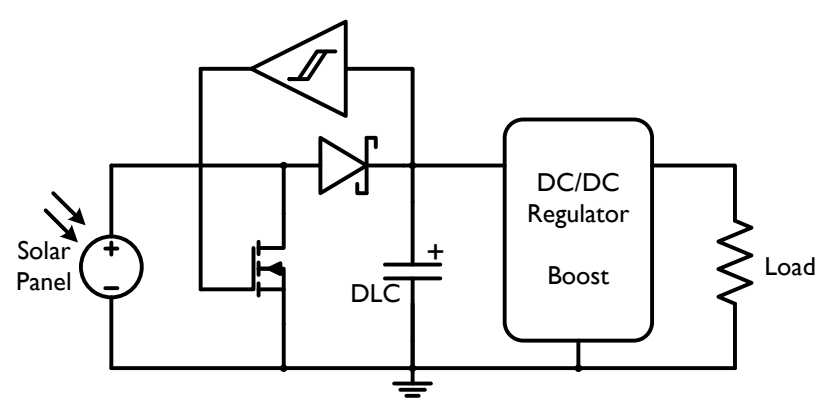

Figure 1. Simplified circuit diagram of the example architecture of a solar energy harvesting system

\section{A. Solar energy harvester architecture}

Fig. 1 depicts the architecture of the solar energy harvesting system used as the basis of the model. In this architecture a double layer capacitor is implemented as energy storage device, which avoids complicated charging mechanisms, but suffers from high self-discharge and low energy density. Therefore these types of storage capacitors can buffer energy only for a short time period and hence need to get replenished regularly. Dimensioning of the DLC capacity is of interest in the system design, since too small capacity leads to reduced availability of the system, while too large capacity increases system size and cost unnecessarily.

Another module with strong influence on the harvester's performance is the solar panel. In this architecture a panel of type $4.5 \mathrm{~V}-100 \mathrm{~mA}$ is implemented. The rather high voltage, due to serial cell-configuration, has the advantage of avoiding the use of an input regulator with boost topology, which comes with losses due to lower efficiencies, especially when input voltage and output current are low. Scaling of the solar panel is desired due to similar reasoning as for the storage element.

Next to their individual behavior, interconnection of solar panel and double layer capacitor plays an important role in available energy levels. In the given architecture these two devices are directly coupled, which not only means that the capacitor is directly charged by the solar input, but also that the DLC voltage influences the operating point of the solar panel. Furthermore, when directly coupled, the capacitor voltage is only limited by the respective opencircuit voltage of the solar panel at given irradiance level. To not endanger the double layer capacitor and to prolong its lifetime, an over-voltage protection circuit consisting of hysteresis comparator and mosfet is part of the architecture.

Maximum Power Point Tracking (MPPT) is not integrated in this architecture, because of the low energy overhead demands in applications this system is used in. However, if necessary, a MPPT subsystem can be integrated into the model easily. 


\section{B. Component models}

Analyzing the individual system components, differentiation can be made between rather static and dynamic behaviors. While the static modules, such as the overvoltage and reverse-current protection, behave similarly over time, the dynamic modules, such as solar panel and double layer capacitor, undergo changes depending on internal and external parameters. For example, the charge and discharge behavior of the storage capacitor is depending on incoming current from the solar panel, current consumption of the load and its own voltage level.

This differentiation is also made in the component modeling. Components with static functions are modeled in an abstractive way, implementing their function rather than their component properties. This allows faster model design without extensive component testing and freedom how to implement the function in the final circuit design. An example of an abstractive function, in this case the over-voltage protection mechanism, is demonstrated in pseudo-code form in Fig. 2. In this example $V_{D L C}$ is the actual voltage of the double layer capacitor, $V_{N O M}$ the nominal voltage of the same and $V_{T H R}$ the hysteresis band of the over-voltage protection. $V_{\text {solar }}$ represents that the solar panel is connected to the double layer capacitor, opposed to be connected to ground, then having a zero terminal voltage.

In contrast, complex components are implement according to an equivalent circuit, representing their electrical behavior. Thereby their behavior is modeled in a more accurate way, but parameter estimation via measurements is unavoidable. Modules with complex component models in this architecture are solar panel and double layer capacitor.

1) Solar panel: A typical way of modeling the electrical behavior of solar panels is the use of the single-diode equivalent circuit, depicted in Fig. 3. Analyzing the voltage and current relations in the circuit, we get the equations

$$
\begin{aligned}
V_{\text {solar }} & =V_{D}-I_{\text {solar }} \cdot R_{s} \\
I_{\text {solar }} & =I_{P h}-I_{D}-I_{s h}
\end{aligned}
$$

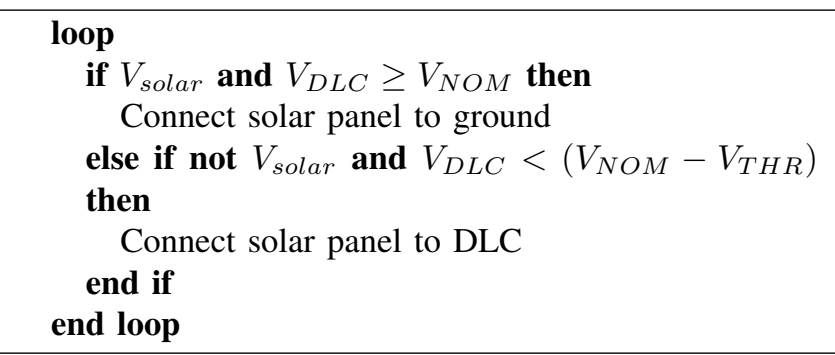

Figure 2. Pseudo-code for the abstractive function of hysteresis overvoltage protection

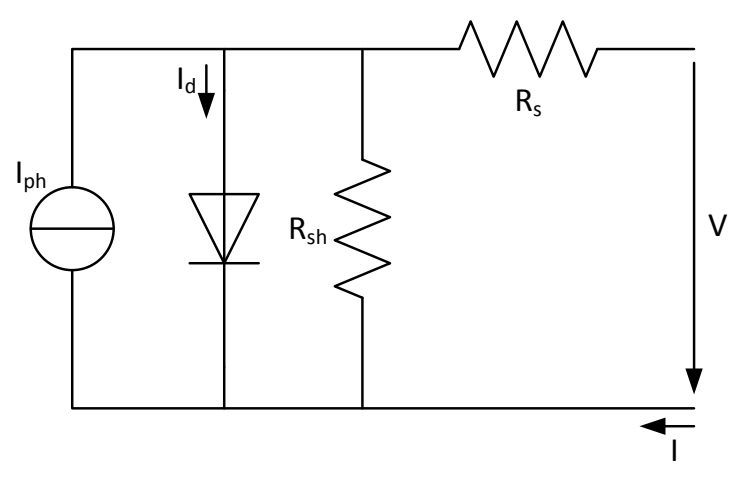

Figure 3. Underlying equivalent circuit of the solar panel model

Substituting (1) in (2) and replacing $I_{D}$ with Shockely's diode equation we get

$$
\begin{aligned}
I_{\text {solar }}= & I_{P h}-I_{0} \cdot\left(e^{\frac{q\left(V_{\text {solar }}+I_{\text {solar }} \cdot R_{s}\right)}{n k T}}-1\right) \\
& -\frac{V_{\text {solar }}+I_{\text {solar }} \cdot R_{s}}{R_{\text {sh }}}
\end{aligned}
$$

which describes solar panel current and voltage in dependency of solar irradiance and temperature. Key parameters for the modeling of the solar panel are the serial resistance $R_{s}$, shunt resistance $R_{s h}$ and the photo-current $I_{P h}$, which can further be described as

$$
I_{P h}=K_{I} \cdot E
$$

Here $E$ is the solar irradiance and $K_{I}$ a factor describing the proportional relationship between irradiance and current.

Different methods to determine these parameters for the single-diode equivalent circuit have been presented in literature and can be found e.g. in [15] and IEC Standard 60891. All these methods use I-V characteristics for the determination process, which is an important classification curve for solar panels. Fig. 5 gives a qualitative overview of variations of the mentioned parameters on the I-V curve of a solar panel.

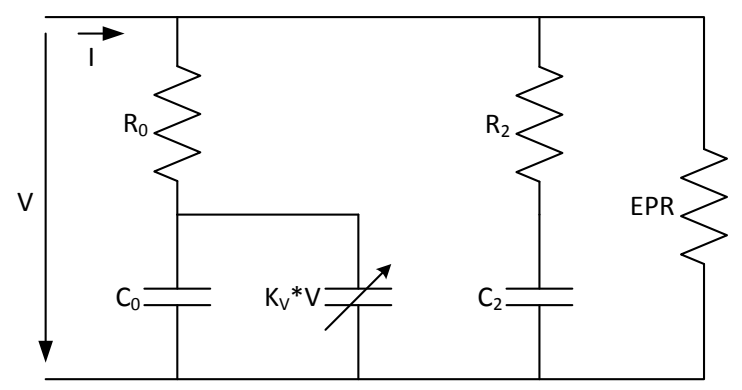

Figure 4. Underlying equivalent circuit of the double layer capacitor 2branch model 


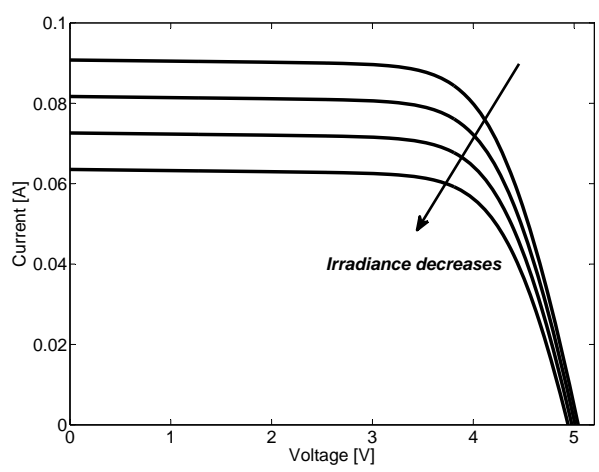

(a)

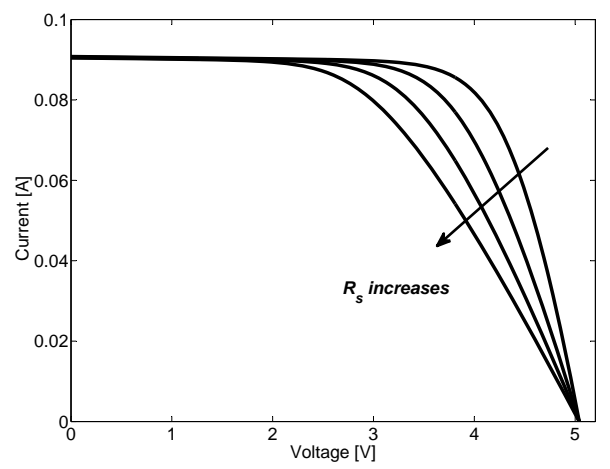

(c)

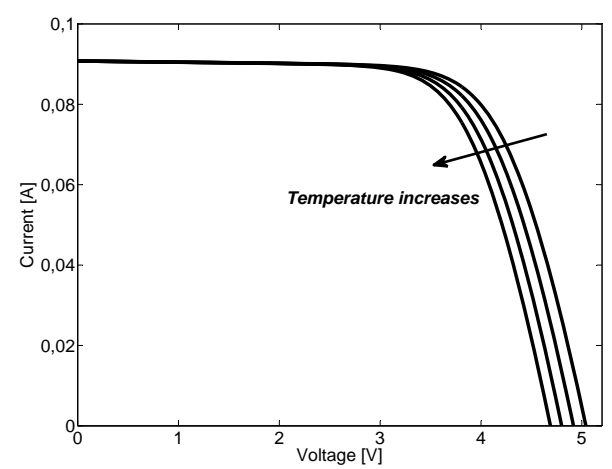

(b)

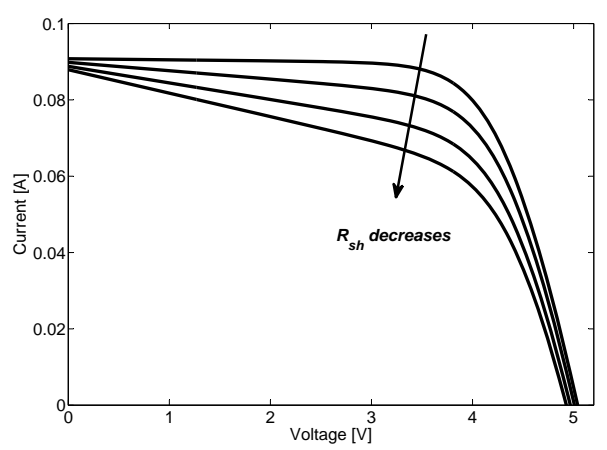

(d)

Figure 5. Influence of model parameters on the I-V curve of a solar panel. Variation of a) Irradiance level, b) Temperature, c) Serial resistance and d) Shunt resistance

2) Double layer capacitor: The double layer capacitor is modeled according to the two-branch model, shown in Fig. 4. This model has been proposed in [10] and includes an immediate branch and a long-term branch, influencing the behavior of the double layer capacitor in short- and long-term respectively. Additionally the model includes an equivalent parallel resistor, representing the self-discharge behavior of the capacitor. Formal description of this model can again be obtained through voltage and current analysis, leading to

$$
\begin{aligned}
& I_{D L C}=I_{1}+I_{2}+I_{3} \\
& V_{D L C}=I_{1} \cdot R_{0}+\int \frac{I_{1}}{C_{0}+K_{V} \cdot V_{D L C}} d t \\
& V_{D L C}=I_{2} \cdot R_{2}+\frac{1}{C_{2}} \int I_{2} d t \\
& V_{D L C}=I_{3} \cdot E P R
\end{aligned}
$$

where $I_{1}, I_{2}$ and $I_{3}$ are the currents through the branches according to Fig. 4 from left to right respectively. In [10] also a method for determination of the parameters $R_{0}, C_{0}$, $R_{2}, C_{2}, K_{V}$ and $E P R$ is given.

\section{System model}

As available energy level, or more specifically the double layer capacitor voltage level, is the main output parameter of the system model, the double layer capacitor itself is the central component. However, the voltage level of the double layer capacitor is influenced by many factors and therefore, connection to other components in the system is of great importance, when evaluating the energy level. Fig. 6 gives an overview of the interactions between components in the model, defining the main operation principle of the system.

Starting at a given time $t_{1}$, the system principle can be described as follows. At time $t_{1}$ the voltage level of the double layer capacitor is $v_{D L C}\left(t_{1}\right)$. This voltage, together with the respective irradiance level $e\left(t_{1}\right)$, sets the operation point of the solar panel, defining the output power of the same according to the known I-V curve. The flowing current $i_{\text {solar }}\left(t_{1}\right)$ will charge the double layer capacitor. At the same time, however, the capacitor gets discharged by the load current $i_{\text {load }}$, which can be approximated as a constant average current, determined by the given sensor node duty cycle, such as

$$
i_{\text {load }}=\delta \cdot i_{\text {active }}+(1-\delta) \cdot i_{\text {idle }}
$$

where $\delta$ is the duty cycle rate $(0<\delta<1)$ and $i_{\text {active }}$ 
and $i_{i d l e}$ are the current levels in active and idle state, respectively. Adding the self-discharge current $i_{\text {leak }}\left(t_{1}\right)$, the resulting charge/discharge current equals to $i_{\text {solar }}\left(t_{1}\right)-$ $i_{\text {load }}-i_{\text {leak }}\left(t_{1}\right)$, changing the charge value of the capacitor to a new value $v_{D L C}\left(t_{2}\right)$, where $t_{2}$ equals to $t_{1}+\Delta t$. With this new voltage level the procedure starts over again.

Additionally to these main components, abstractive modules influence the system behavior in the model. These include, as mentioned before, the over-voltage protection, reverse-current protection and voltage conversion for the load system. Furthermore, other abstractive functions (e.g., a MPPT module) can be easily added.

\section{EXPERIMENTAL SETUP}

When it comes to experiment conduction with the model, distinction can be made between two cases, namely model evaluation and system simulation. In model evaluation the component and system models are validated against a measured reference to show the accuracy and satisfaction of the modeling. After model evaluation, the model can be used to simulate system behavior in different situations and with different dimensioning parameters. For both experiments the system model, i.e. all component models and connections, has been implemented with Matlab/Simulink. This environment was chosen due to availability and the possibility to implement both, electrical and abstractive functions with support of predefined libraries. Simulink also provides flexibility towards extension and modification of implemented models, and therefore allows changes in an easy fashion.

\section{A. Model evaluation}

Subsystems of the energy harvesting model have been validated individually with laboratory measurements. For the evaluation of the complete model, a comparison between simulation outcome and an actual deployment has been carried out. The deployment took place in Sundsvall, Sweden in

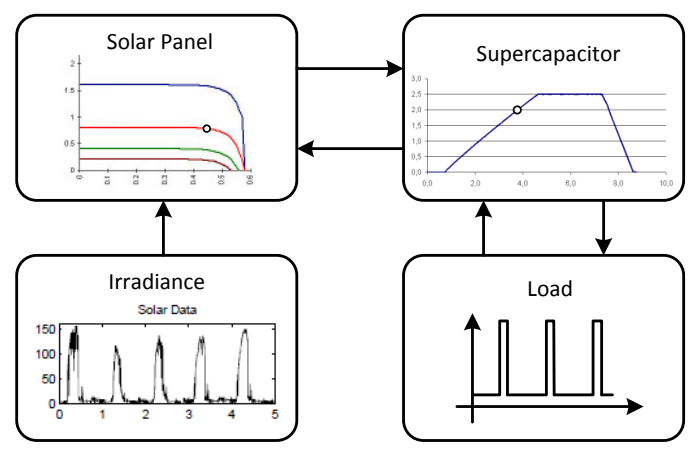

Figure 6. Basic overview of the interaction between main modules in the system model
Table I

DIMENSIONING PARAMETERS OF THE PRESENTED SOLAR ENERGY HARVESTING ARCHITECTURE

\begin{tabular}{ll}
\hline Parameter & Main constraints \\
\hline Solar panel size & Cost, size \\
DLC capacity & Cost, size \\
Load consumption & Application requirements \\
Solar irradiation & Location of deployment \\
Load availability & Application requirements \\
Hysteresis band & DLC lifetime \\
\hline
\end{tabular}

the winter 2009/2010 (i.e., November to February). Same architecture as previously described was implemented in both hardware and the simulation model. Hardware configuration of the main components in the system were a $4.5 \mathrm{~V}-100 \mathrm{~mA}$ solar panel of size $94 \times 61 \mathrm{~mm}^{2}$, double layer capacitors [16] with both, $10 \mathrm{~F}$ and $22 \mathrm{~F}$ capacity, as well as a duty-cycled wireless sensor load [17].

The systems were placed spatially close to a reference sensor [18], measuring solar irradiance levels in a fiveminute interval. The gained data on solar irradiance has afterwards been used as input parameter for the simulation.

More information on hardware configuration, deployment site and measurement results can be found in [1].

\section{B. Simulations}

After model evaluation, the model has been used to carry out simulations, showing its suitability for addressing dimensioning problems. Influence from variations in main system parameters, such as solar panel size, load consumption and DLC capacity are investigated. A complete list of dimensioning parameters for the used architecture is given in Table I.

The same system architecture and implementation as in the model evaluation has been used to conduct these simulations. Furthermore, solar irradiance input for the simulations are taken from data, measured by a weather station in Sundsvall, Sweden.

Several simplification assumptions have been made for the simulations. These include ideal scalability of solar panels, meaning that we simplify a solar panel of $x$-times the size of the reference panel will provide same voltage behavior, but $x$-times the current output at same irradiance levels. Furthermore, we specify the system as not functioning at times when the double layer capacitor voltage is lower than the minimum input voltage of the implemented boost converter [19]. In this periods of time, the consumption of the load system is assumed to be zero.

\section{Results}

For general system behavior description, two system states can be distinguished, namely charging and discharging state. If solar irradiance at a certain time $t$ is high enough to lead 


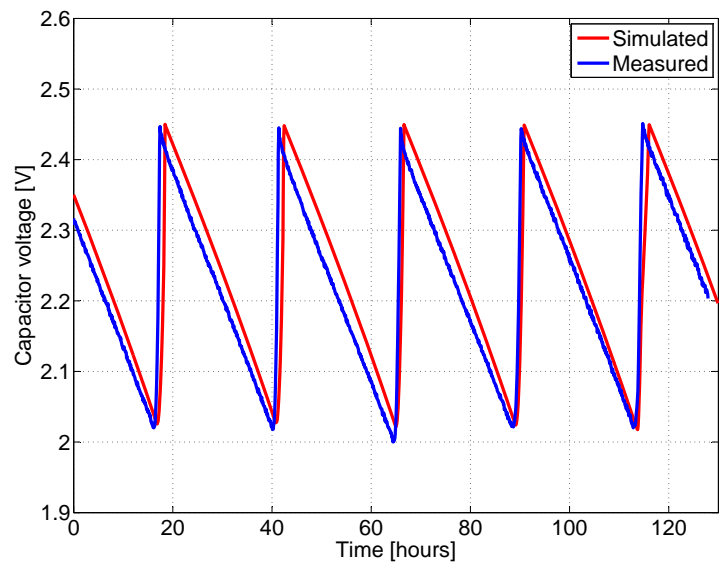

(a)

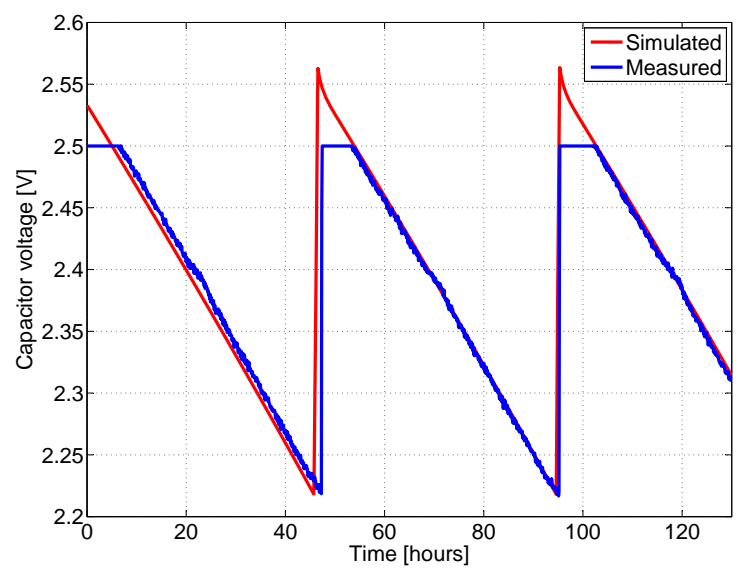

(b)

Figure 7. Comparison between energy levels from simulation and deployment for a system with a) 10 Farad DLC and b) 22 Farad DLC

to a positive charging condition as described in section III-C, the capacitor will be charged until its voltage indicates that full charge level is reached. Charging might precociously stop when solar irradiance changes to a level, leading to negative charge condition. In the former case, discharge current is only defined by self-discharge rate of the double layer capacitor used and the power consumption of the load system. However, when charging was precociously stopped, this discharge rate might be reduced due to incoming solar energy. Has charge state been stopped because of full capacitor charge, two conditions have to be fulfilled to start recharging. The incoming irradiance level has to be high enough to lead to a positive charge level and the capacitor has to have been discharged to a low enough voltage, allowing recharge (i.e. lower hysteresis bond of the overvoltage protection). If charging was stopped precociously, only the irradiance condition influences recharging.

Fig. 7 shows an excerpt of the evaluation results of the system model. For this evaluation both, a $10 \mathrm{~F}$ DLC and $22 \mathrm{~F}$ DLC, were implemented in the model and compared to energy levels, obtained in deployment of each system respectively. In both cases, good conformance between measured and simulated data can be observed. The described system behavior can be monitored in all cases and effects of this behavior on differences between different system configurations can be seen. Due to higher energy saved in the $22 \mathrm{~F}$ DLC and the resulting slower voltage decrease, its system has a charge-discharge cycle of two days, while the $10 \mathrm{~F}$ system shows a daily cycle.

In Fig. 7a a time-offset between measured and simulated data can be observed occasionally. This mismatch occurs due to small variation of lower hysteresis bond in the deployed system (analog comparator), while the lower hysteresis bond in the model is stable (abstractive module). Missing of the tip in the measured data series in $7 b$ is caused by the measurement setup, which was limited to a measurement range between $0 \mathrm{~V}$ and $2.5 \mathrm{~V}$. This lead to full-scale ADC reading also for higher energy levels.

After validation of the system model, influence of component parameter variations have been investigated. As mentioned before, solar panel size, DLC capacity and load consumption are the main influencing parameters in the harvesting system. Especially decisions on solar panel size and DLC capacity for given load current and irradiance level constraints are of interest. However, some other parameter, as described in Table I, can be used to further optimize the dimensioning of the system.

Fig. 8 gives an exemplary relation between these parameters for the given architectural model. For these cases solar panel size has been swept according to the simplification made in IV-B, while the maximum current consumption of the load, fulfilling the given availability restriction of the system, was determined. All simulations use the same irradiance data of December 2009 in Sundsvall, Sweden, which was the month with lowest solar irradiance in the year (i.e., analyzing worst case scenario). Additionally to the solar size in Fig. 8a differences for varying DLC capacity are shown, while in Fig. 8b influence of different availability requirements is presented. Last, in Fig. 8c, the hysteresis band of the over-voltage protection circuit is varied. In all simulations, excluding Fig. 8b, where availability is varied, determination of maximum load consumption is based on a $100 \%$ availability requirement.

The data shows, that generally increasing the solar panel size leads to a higher maximum load current under otherwise same conditions. However, systems with a given DLC capacity, show saturation effects, which means augmentation of solar panel size from a certain point onward has no impact on heightening the allowed load consumption anymore. This can be explained due to the fact, that even when fully 


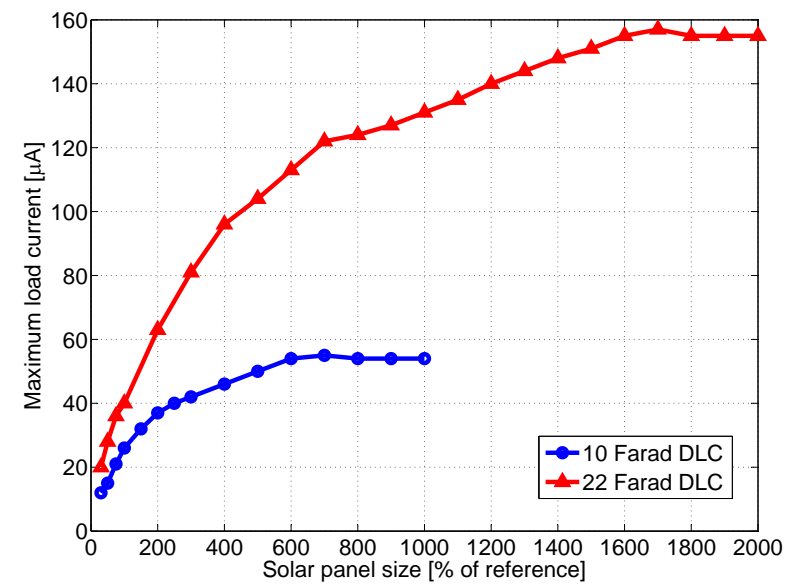

(a)

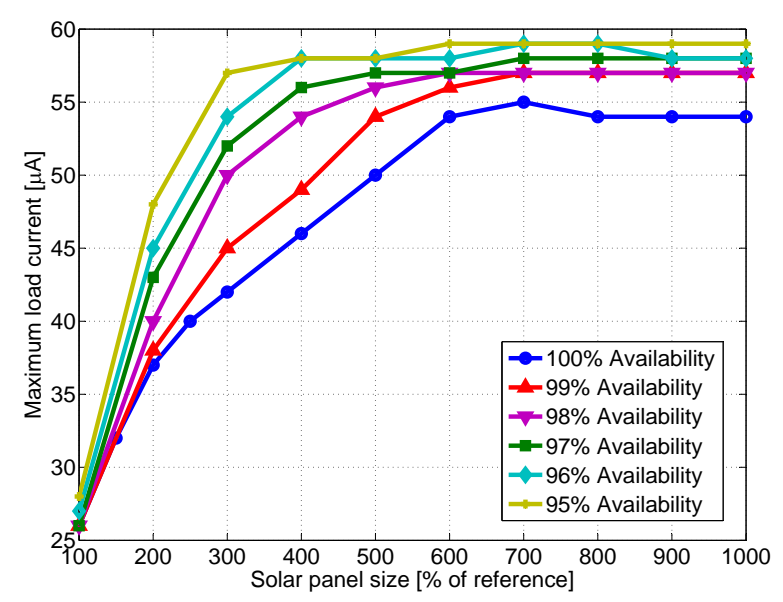

(b)

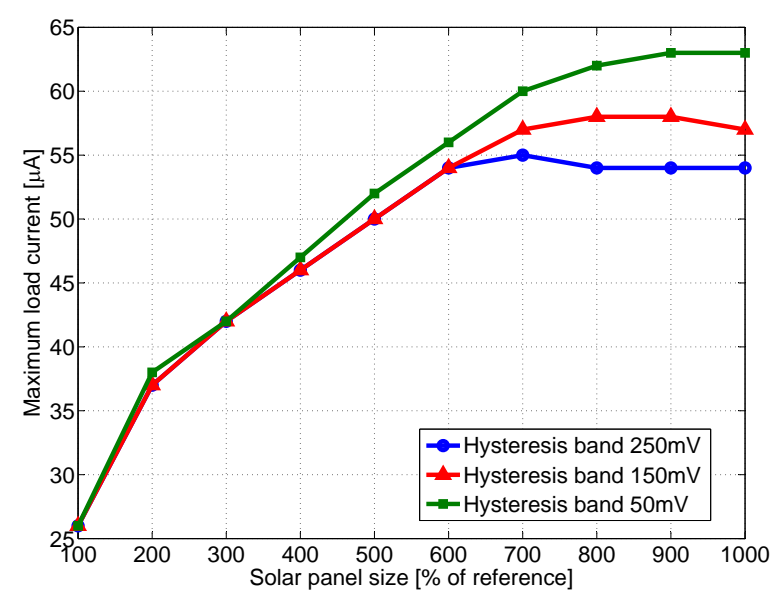

(c)

Figure 8. Influence of dimensioning parameter variation on maximum load current. For different a) double layer capacitor (constants: 100\% availability; $350 \mathrm{mV}$ hysteresis), b) availability levels (constants: $10 \mathrm{~F}$ capacity; $350 \mathrm{mV}$ hysteresis) and c) over-voltage hysteresis settings (constants: 100\% availability; $10 \mathrm{~F}$ capacity) charged, the capacitor does not provide enough energy to endure times without solar energy availability. As increased solar panel size factorizes charge current, but not DLC capacity, influence of solar panel size will stop from a certain size onwards. Furthermore, increasing the storage capacitor by a factor of 2.2 does not lead to a constant increase in maximum load current by the same factor. Nonetheless, in all simulated cases the maximum allowed load consumption is higher in case of larger DLC. The factors of increased load consumption lay between 1.5 and 2.8 for same solar panel size respectively and are rising with increasing solar panel size. One probable reason of this factor variation results from different self-discharge rates between the two capacitors and the ratio of self-discharge rate over load current.

Further on, availability can be used as a trade off factor, when the application allows limited downtime of network nodes. Not surprisingly, reducing availability demands leads to higher allowed load consumption. However, according to Fig. $8 \mathrm{~b}$ an area of maximum gain can be identified. This is, because sensitivity to solar panel increase weakens with increasing solar panel size.

Fig. $8 \mathrm{c}$ depicts, that lowering the hysteresis band of the over-voltage protection circuit, used in the example architecture, leads to an increase in maximum load current. This effect has its reason in timing problems, occurring due to hysteresis. Since the hysteresis brings a second charge condition, the capacitor might not always be fully charged in the end of a light-period and thereby cannot endure the maximum dark-period length, the capacitors capacity would allow. By reducing the hysteresis band, the impact of this timing problem is reduced. However, the stress on the DLC will increase at the same time as it will be encounter more charge/discharge cycles. The effect shows more with higher solar panel sizes as charge time in that case is reduced.

\section{CONCLUSIONS}

Dimensioning is a crucial task in any electronic system to fit application constraints. Since micro-scale solar harvesting systems are tremendously application and location dependent, a method for carrying out the dimensioning process of these systems effectively is required.

In this paper we presented such a method for system dimensioning, based on simulation of available energy levels. A hybrid system modeling approach was presented, allowing accurate simulation of available energy, depending on solar irradiance level input and load consumption. The presented model has been evaluated in laboratory setups and against real-world deployment of the same system architecture. Further on, the model was used to target dimensioning problems of key parameters, such as solar panel size and energy storage capacity in respect to constraints of load current consumption, availability demands and solar irradiation conditions. The presented method showed its capability of 
supporting parameter optimization tasks and is therefore a suitable method for addressing system dimensioning.

For the presented system example, the model showed relations between key system parameters, enabling to make decisions on component choices under known application requirements. Hence the model prevents excessive system testing or undesired results from miss-dimensioning, such as reduced availability or higher size and cost than needed. Also impact of trade-off factors, such as slightly reduced availability constraints and hysteresis level adjustments were shown.

Radiation condition input to the simulator is kept in a flexible way. Because solar energy harvesting systems are susceptible to environmental conditions, such as changing weather conditions, accurate input data for the simulator is important for the performance of the dimensioning output. While in this case historical data was used, this might not always be available for a desired deployment location. Predicted input data can be used in these cases, however, usually including some prediction error.

\section{ACKNOWLEDGMENT}

Financial support of the project Sensible Things that Communicate by KK-foundation is gratefully acknowledged.

\section{REFERENCES}

[1] S. Bader and B. Oelmann, "Enabling battery-less wireless sensor operation using solar energy harvesting at locations with limited solar radiation," in Fourth International Conference on Sensor Technologies and Applications, SENSORCOMM'10, 2010.

[2] V. Raghunathan, A. Kansal, J. Hsu, J. Friedman, and M. Srivastava, "Design considerations for solar energy harvesting wireless embedded systems," in Proceedings of the 4th international symposium on Information processing in sensor networks, 2005, pp. $457-462$.

[3] F. Simjee and P. Chou, "Everlast: long-life, supercapacitoroperated wireless sensor node," in Proceedings of the 2006 international symposium on Low power electronics and design, 2006, pp. 197 - 202.

[4] C. Park and P. Chou, "Ambimax: Autonomous energy harvesting platform for multi-supply wireless sensor nodes," in 3rd Annual IEEE Communications Society on Sensor and Ad Hoc Communications and Networks, 2006, pp. 168-177.

[5] X. Jiang, J. Polastre, and D. Culler, "Perpetual environmentally powered sensor networks," in Proceedings of the 4th international symposium on Information processing in sensor networks, 2005, pp. 463-468.

[6] W. De Soto, S. A. Klein, and W. A. Beckman, "Improvement and validation of a model for photovoltaic array performance," Solar Energy, vol. 80, no. 1, pp. 78-88, Jan. 2006.
[7] D. Brunelli, D. Dondi, a. Bertacchini, L. Larcher, P. Pavan, and L. Benini, "Photovoltaic scavenging systems: Modeling and optimization," Microelectronics Journal, vol. 40, no. 9, pp. 1337-1344, Sep. 2009.

[8] W. Xiao, W. G. Dunford, and A. Capel, "A novel modeling method for photovoltaic cells," in IEEE 35th Annual Power Electronics Specialists Conference, 2004, pp. 1950-1956.

[9] J. Jeong, "A practical theory of micro-solar power sensor networks," Ph.D. dissertation, EECS Department, University of California, Berkeley, Apr 2009. [Online]. Available: http://www.eecs.berkeley.edu/Pubs/TechRpts/2009/EECS2009-49.html

[10] R. Faranda, M. Gallina, and D. T. Son, "A new simplified model of double-layer capacitors," in International Conference on Clean Electrical Power, 2007, pp. 706-710.

[11] A. S. Weddell, G. V. Merrett, N. R. Harris, and N. M. White, "Energy devices for sensor networks: Properties for simulation and deployment," in 1st International Conference on Wireless Communication, Vehicular Technology, Information Theory and Aerospace \& Electronic Systems Technology, 2009, pp. 26-30.

[12] D. Krüger, C. Buschmann, and S. Fischer, "Solar Powered Sensor Network Design and Experimentation," in 6th International Symposium on Wireless Communication Systems, 2009, pp. 11-15.

[13] J. W. Kimball, B. T. Kuhn, and R. S. Balog, "A System Design Apporach for Unattended Solar Energy Harvesting Supply," IEEE Transactions on Power Electronics, vol. 24, no. 4, pp. 952-962, 2009.

[14] J. Taneja, J. Jeong, and D. Culler, "Design, modeling, and capacity planning for micro-solar power sensor networks," in Proceedings of the 7th international conference on Information processing in sensor networks, 2008, pp. 407-418.

[15] A. Aberle, S. Wenham, and M. Green, "A new method for accurate measurements of the lumped series resistance of solar cells," in Conference Record of the Twenty Third IEEE Photovoltaic Specialists Conference, 1993, pp. 133-139.

[16] PowerStor Supercapacitors - B Series (BU-SB08841), Cooper Bussmann, 2008.

[17] (2010) Sentio series of sensor nodes - website. [Online]. Available: http://www.miun.se/stc/Sentio

[18] Solar Radiation Sensor 7821, Davis Instruments, 2000.

[19] TPS6107x datasheet (SLVS510C), Texas Instruments, 2009. 